Research Article

\title{
The Generalized Inverse Generalized Weibull Distribution and Its Properties
}

\author{
Kanchan Jain, Neetu Singla, and Suresh Kumar Sharma \\ Department of Statistics, Panjab University, Chandigarh 160014, India \\ Correspondence should be addressed to Kanchan Jain; jaink14@gmail.com
}

Received 25 February 2014; Revised 2 July 2014; Accepted 8 July 2014; Published 6 August 2014

Academic Editor: Farrukh Mukhamedov

Copyright (c) 2014 Kanchan Jain et al. This is an open access article distributed under the Creative Commons Attribution License, which permits unrestricted use, distribution, and reproduction in any medium, provided the original work is properly cited.

\begin{abstract}
The Inverse Weibull distribution has been applied to a wide range of situations including applications in medicine, reliability, and ecology. It can also be used to describe the degradation phenomenon of mechanical components. We introduce Inverse Generalized Weibull and Generalized Inverse Generalized Weibull (GIGW) distributions. GIGW distribution is a generalization of several distributions in literature. The mathematical properties of this distribution have been studied and the mixture model of two Generalized Inverse Generalized Weibull distributions is investigated. Estimates of parameters using method of maximum likelihood have been computed through simulations for complete and censored data.
\end{abstract}

\section{Introduction}

The Generalized Weibull (GW) distribution possessing bathtub failure rate was introduced by Mudholkar and Srivastava [1]. Mudholkar et al. [2] and Mudholkar and Hutson [3] applied GW distribution for analysis of data relating to bus motor failure, head and neck cancer, and flood.

Inverse distributions, namely, Inverse Gamma, Inverse Generalized Gamma, Inverse Weibull, and Inverse Rayleigh, have been studied in literature [4-8]. Keller et al. [9] studied shapes of density and failure rate function for the basic inverse model and Drapella [6] worked on Inverse Weibull (IW) distribution. Drapella [6] and Mudholkar and Kolia [10] suggested the names complementary Weibull and reciprocal Weibull. These distributions have applications in reliability engineering and medical sciences and are used for modelling infant mortality, wear-out periods, degradation of mechanical components [11], times of breakdown of an insulating fluid subject to the action of constant tension [5], and loadstrength relationship for a component [12]. Aleem and Pasha [13] studied some distributional properties of IW.

In this paper, we first introduce a three-parameter continuous probability distribution on the positive real line, known as Inverse Generalized Weibull (IGW) distribution. It is the distribution of reciprocal of a variable distributed according to the Generalized Weibull distribution. It can also be called Complementary or Reciprocal Generalized Weibull distribution. Using IGW distribution, a four-parameter distribution named as Generalized Inverse Generalized Weibull (GIGW) distribution is introduced and its properties are studied. The mixture of two GIGW distributions has been investigated. Empirical estimates of parameters have been found using maximum likelihood method for complete and censored data. An application to real data set has been provided to illustrate the potentiality of the proposed models. The paper is organized as follows.

In Section 2, we introduce the Inverse Generalized Weibull (IGW) and Generalized Inverse Generalized Weibull (GIGW) distributions. Moments of GIGW distribution have been derived in Section 2.1. A mixture of two GIGW distributions and its properties have been discussed in Section 3. In Section 4 , the nonlinear equations for finding the maximum likelihood estimates of the parameters have been derived for both uncensored and censored case. To check the theoretical results, simulations have been carried out in Section 5 and an application to a real data set has been provided. In case of censoring, the elements of the information matrix are included in the appendix for estimation and testing purposes. 


\section{The Generalized Inverse Generalized Weibull (GIGW) Distribution}

The cumulative distribution function (cdf) and the probability density function (pdf) of GW distribution [1] are

$$
\begin{gathered}
G_{\mathrm{GW}}(x)=\left(1-e^{-(\lambda x)^{\beta}}\right)^{\alpha}, \quad x>0, \alpha>0, \beta>0, \lambda>0, \\
g_{\mathrm{GW}}(x)=\alpha \beta \lambda^{\beta} x^{\beta-1}\left(1-e^{-(\lambda x)^{\beta}}\right)^{\alpha-1} e^{-(\lambda x)^{\beta}} \\
x>0, \alpha>0, \beta>0, \lambda>0
\end{gathered}
$$

respectively.

For introducing GIGW distribution, we first propose Inverse Generalized Weibull (IGW) distribution with cdf and pdf written as

$$
\begin{gathered}
G_{\mathrm{IGW}}(x)=1-\left(1-e^{-(\lambda / x)^{\beta}}\right)^{\alpha}, \\
g_{\mathrm{IGW}}(x)=\alpha \lambda^{\beta} \beta e^{-(\lambda / x)^{\beta}} x^{-(\beta+1)}\left(1-e^{-(\lambda / x)^{\beta}}\right)^{\alpha-1},
\end{gathered}
$$

where $x>0$ and $\alpha, \beta, \lambda>0$.

The corresponding survival and hazard rate functions are given by

$$
\begin{gathered}
\bar{G}_{\mathrm{IGW}}(x)=\left(1-e^{-(\lambda / x)^{\beta}}\right)^{\alpha}, \\
h_{\mathrm{IGW}}(x)=\alpha \lambda^{\beta} \beta e^{-(\lambda / x)^{\beta}} x^{-(\beta+1)}\left(1-e^{-(\lambda / x)^{\beta}}\right)^{-1},
\end{gathered}
$$

respectively.

A generalization of IGW distribution is proposed as Generalized Inverse Generalized Weibull (GIGW) distribution with cdf

$$
F_{\mathrm{GIGW}}(x)=1-\left(1-e^{-\gamma(\lambda / x)^{\beta}}\right)^{\alpha}, \quad x>0,
$$

where $\lambda>0$ is the scale parameter and $\alpha, \beta$, and $\gamma>0$ are shape parameters.

This gives the density function as

$$
\begin{array}{r}
f_{\mathrm{GIGW}}(x)=\alpha \lambda^{\beta} \beta \gamma x^{-(\beta+1)} e^{-\gamma(\lambda / x)^{\beta}}\left(1-e^{-\gamma(\lambda / x)^{\beta}}\right)^{(\alpha-1)}, \\
x>0 .
\end{array}
$$

By substituting

$$
u=-\left(1-e^{-\gamma(\lambda / x)^{\beta}}\right)^{\alpha}
$$

it can be easily shown that (7) is a density function.

The survival and hazard rate functions of GIGW distribution have the forms

$$
\begin{gathered}
\bar{F}_{\mathrm{GIGW}}(x)=\left(1-e^{-\gamma(\lambda / x)^{\beta}}\right)^{\alpha}, \\
h_{\mathrm{GIGW}}(x)=\alpha \lambda^{\beta} \beta \gamma x^{-(\beta+1)} e^{-\gamma(\lambda / x)^{\beta}}\left(1-e^{-\gamma(\lambda / x)^{\beta}}\right)^{-1},
\end{gathered}
$$

respectively.
The plots of density function are displayed in Figure 1 for different values of the parameters. In this figure, the plots for $\gamma=1$ correspond to IGW distribution whereas the other two plots for $\gamma<1$ and $>1$ depict the pdfs of GIGW distribution.

It is depicted by Figure 1 that IGW $(\gamma=1)$ and GIGW are a positively skewed distribution.

The hazard rate functions of IGW $(\gamma=1)$ and GIGW distributions are plotted in Figure 2 for different combinations of parametric values.

Figure 2 indicates that

(i) for $\alpha, \beta, \lambda>1$,

(a) hazard rate function for IGW distribution lies in between the hazard rate functions of GIGW distribution when $\gamma$ is less than or greater than 1 ;

(b) for $\gamma<1$, hazard rate of GIGW distribution decreases at a faster rate than that of IGW distribution;

(ii) for $\alpha, \beta, \lambda<1$,

(a) hazard rate function for IGW distribution lies below the hazard rate functions of GIGW distribution when $\gamma$ is less than or greater than 1 ;

(b) hazard rate of GIGW distribution decreases at a faster rate than that of IGW distribution.

If $X$ is a random variable (r.v.) with pdf given by (7), we use the notation $X \sim \operatorname{GIGW}(\lambda, \alpha, \beta, \gamma)$.

Special Cases. (1) When $\alpha=1$, Generalized Inverse Generalized Weibull (GIGW) reduces to Generalized Inverse Weibull (GIW) [14] with shape parameters $\beta$ and $\gamma$ and scale parameter $\lambda$.

(2) Putting $\gamma=1$, we get IGW as a particular case of GIGW.

(3) $\alpha=1$ and $\gamma=1$ give Inverse Weibull (IW) distribution with parameters $\lambda$ and $\beta$.

Remark 1. Observations from $\operatorname{GIGW}(\lambda, \alpha, \beta, \gamma)$ distribution can be simulated using the transformation

$$
X=\lambda \gamma^{1 / \beta}\left[\log \left(1-(1-U)^{1 / \alpha}\right)\right]^{-1 / \beta},
$$

where $U$ follows Uniform distribution on $(0,1)$.

We simulate a data set from GIGW distribution with parameters $\lambda=0.05, \alpha=5, \beta=2$, and $\gamma=0.3$ and plot the exact and empirical cdfs of GIGW in Figure 3 to check the correctness of procedure for simulations.

The closeness of two cdfs implies the validity of the procedure for generating observations from GIGW.

2.1. Moments of GIGW Distribution. Moments of a distribution are important characteristics of any distribution. These are helpful in finding average, dispersion, coefficients of skewness, and kurtosis. 


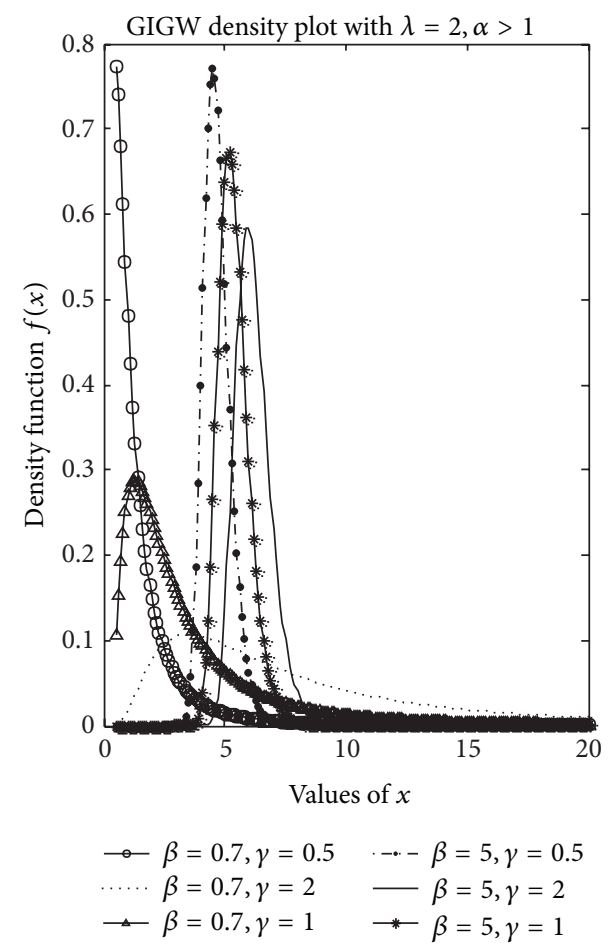

(a)

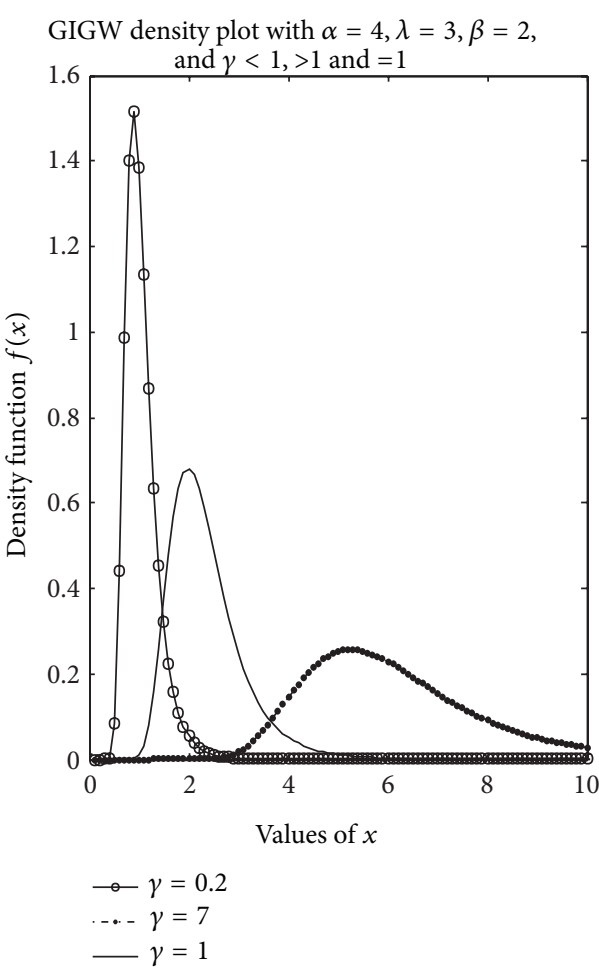

(c)

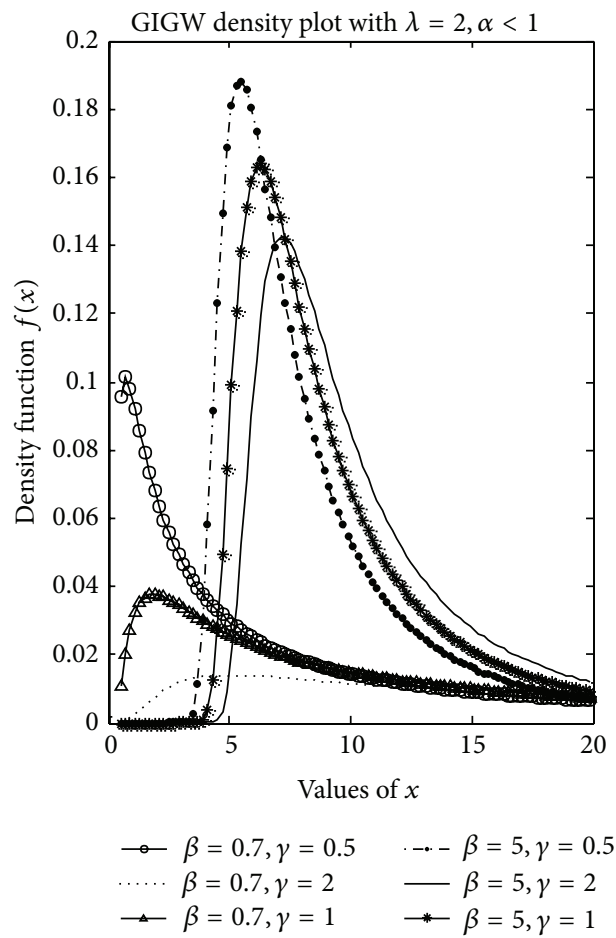

(b)

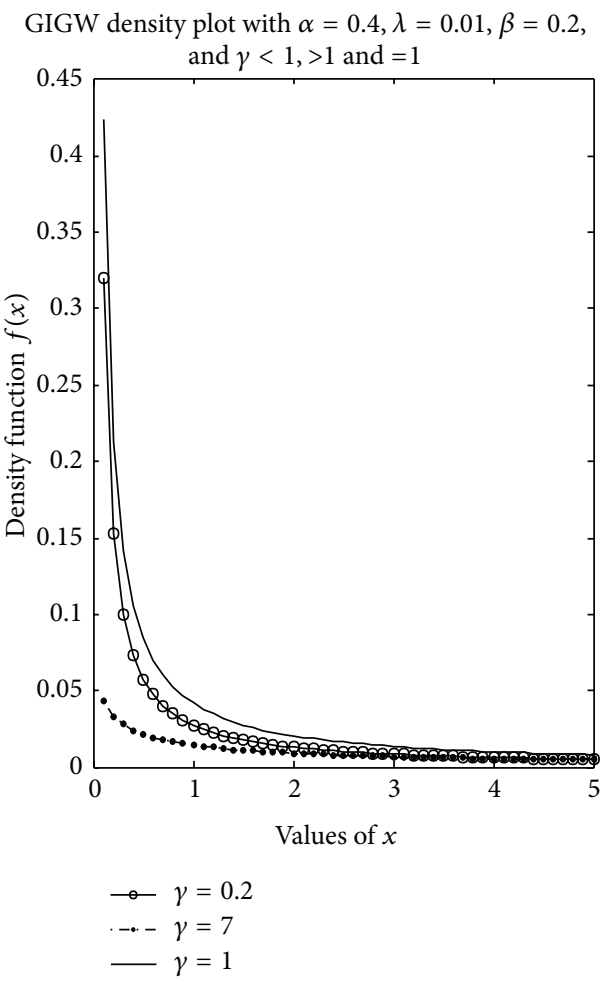

(d)

Figure 1: Plots for GIGW density function. 

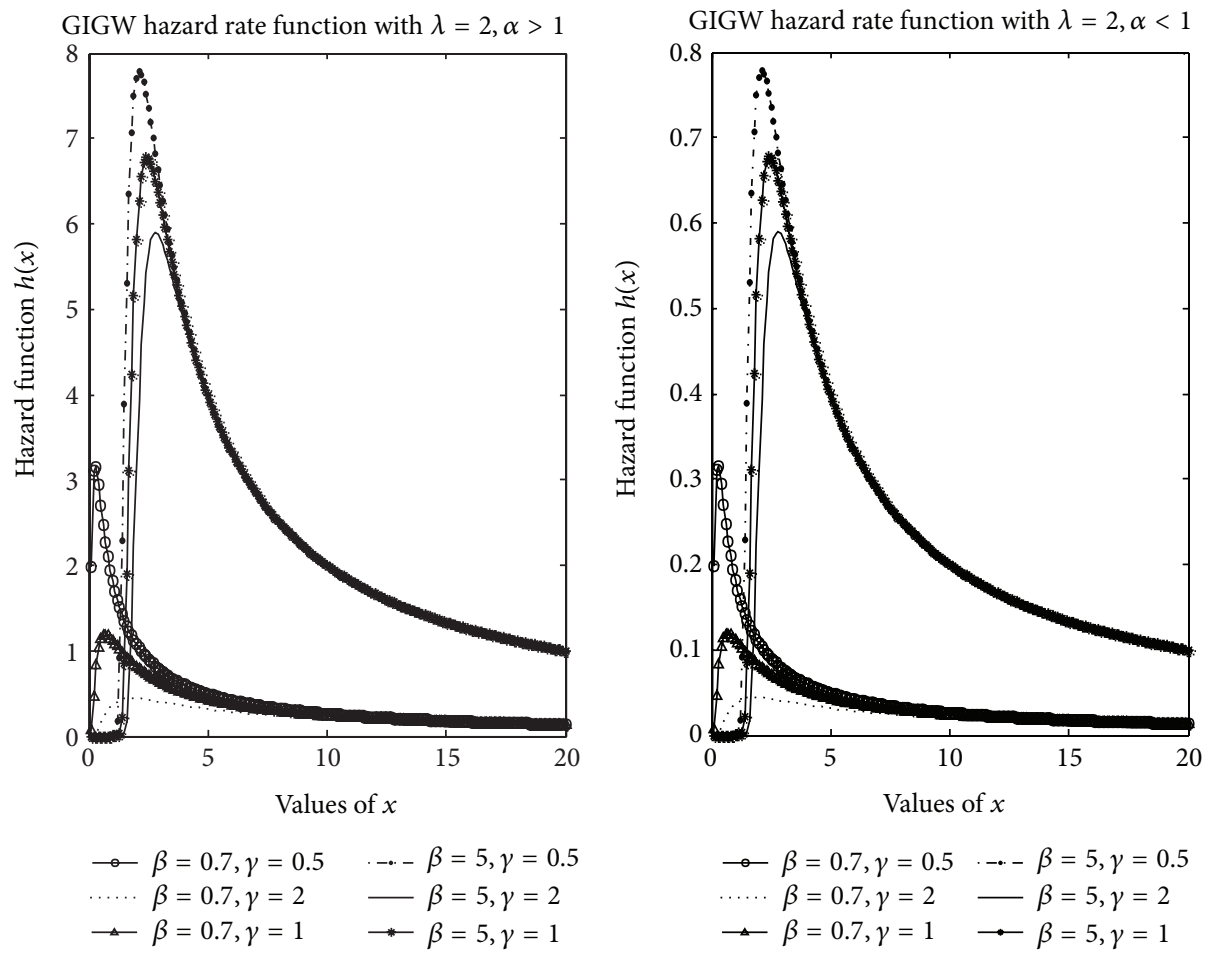

$$
\begin{array}{llrl}
\rightarrow \beta & =0.7, \gamma=0.5 & \rightarrow-\beta & =5, \gamma=0.5 \\
\cdots \beta & =0.7, \gamma=2 & -\beta & =5, \gamma=2 \\
\rightarrow \beta & =0.7, \gamma=1 & *-\beta & =5, \gamma=1
\end{array}
$$

(a)

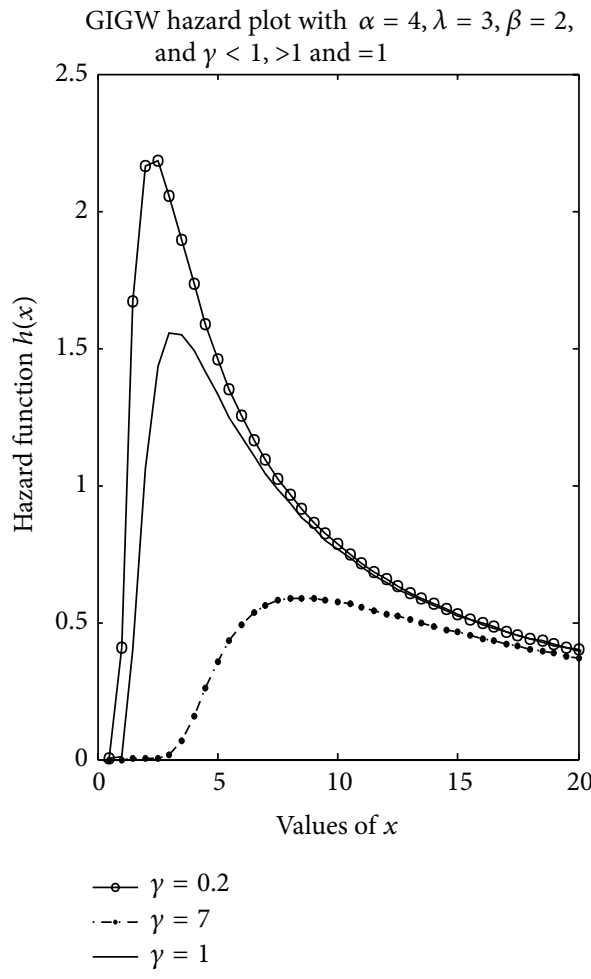

(c)
GIGW hazard plot with $\alpha=0.4, \lambda=0.01, \beta=0.2$,

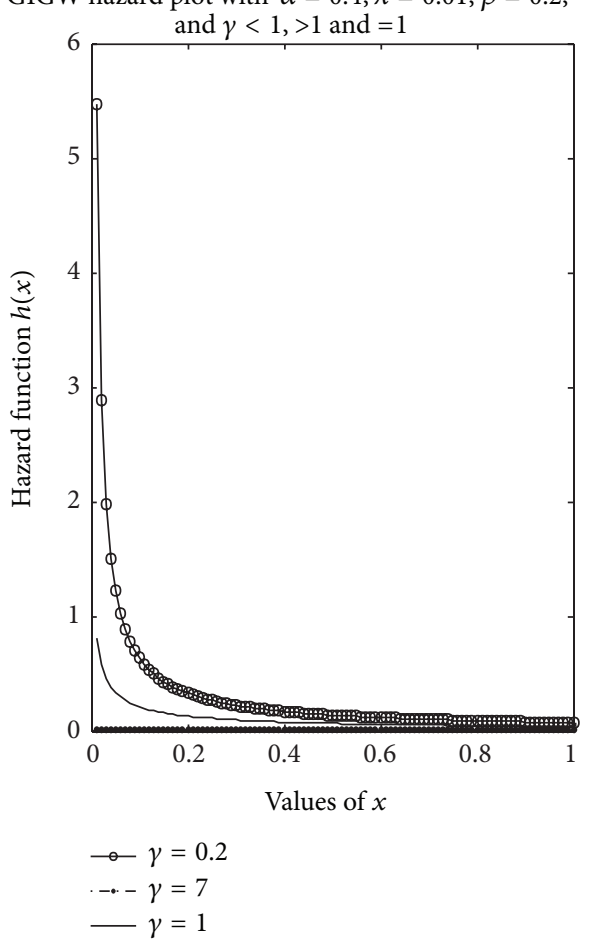

(d)

Figure 2: Plots for GIGW hazard function. 


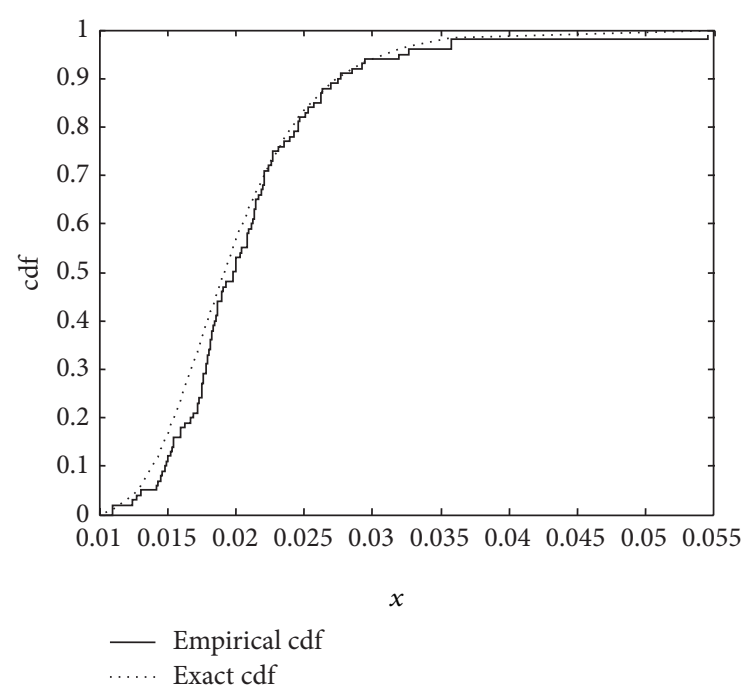

FIgURE 3: Plots for comparison of exact and empirical cdfs of GIGW.

For $X \sim \operatorname{GIGW}(\lambda, \alpha, \beta, \gamma)$, the $k$ th order moment around zero is written as

$$
\begin{aligned}
E\left(X^{k}\right)=\int_{0}^{\infty} & x^{k} \alpha \gamma \beta \lambda^{\beta} x^{-(\beta+1)} e^{-\gamma(\lambda / x)^{\beta}} \\
& \times\left(1-e^{-\gamma(\lambda / x)^{\beta}}\right)^{\alpha-1} d x .
\end{aligned}
$$

Taking $\gamma(\lambda / x)^{\beta}=y$,

$$
\begin{aligned}
E\left(X^{k}\right) & =\alpha \lambda^{k} \gamma^{k / \beta} \int_{0}^{\infty} y^{-k / \beta} e^{-y}\left(1-e^{-y}\right)^{\alpha-1} d y \\
& =\alpha \lambda^{k} \gamma^{k / \beta} \int_{0}^{\infty} y^{-k / \beta} e^{-y}\left(1+\sum_{i=1}^{\infty} a_{i} e^{-y i}\right) d y,
\end{aligned}
$$

using binomial expansion with $a_{i}=\left((-1)^{i}(\alpha-1)(\alpha-2) \cdots(\alpha-\right.$ i))/i!.

Hence

$$
E\left(X^{k}\right)=\alpha \lambda^{k} \gamma^{k / \beta} \Gamma\left(1-\frac{k}{\beta}\right)\left[1+\sum_{i=1}^{\infty} \frac{a_{i}}{(i+1)^{1-(k / \beta)}}\right]
$$

gives the general expression for $k$ th moment of GIGW distribution.

Remark 2. For $\alpha=1$ in (14), we get the $k$ th moment of GIW distribution given by de Gusmão et al. [14]

The moment generating function (mgf) of $X$ can be written as

$M(t)=\alpha \sum_{k=0}^{n}\left[\frac{t^{k}}{k !} \lambda^{k} \gamma^{k / \beta} \Gamma\left(1-\frac{k}{\beta}\right)\left[1+\sum_{i=1}^{\infty} \frac{a_{i}}{(i+1)^{1-(k / \beta)}}\right]\right]$,

for $|t|<1$.
The cumulant generating function of $X$ is given by

$$
\begin{aligned}
& K(t)=\log [M(t)] \\
&=\log \left\{\alpha \sum _ { k = 0 } ^ { n } \left[\frac{t^{k}}{k !} \lambda^{k} \gamma^{k / \beta} \Gamma\left(1-\frac{k}{\beta}\right)\right.\right. \\
&\left.\left.\times\left[1+\sum_{i=1}^{\infty} \frac{a_{i}}{(i+1)^{1-(k / \beta)}}\right]\right]\right\}, \\
& \text { for }|t|<1 .
\end{aligned}
$$

Using (14), the mean and variance of GIGW can be written as

$$
\begin{aligned}
& E(X)=\alpha \lambda \gamma^{1 / \beta} \Gamma\left(1-\frac{1}{\beta}\right)\left[1+\sum_{i=1}^{\infty} \frac{a_{i}}{(i+1)^{1-(1 / \beta)}}\right], \\
& V(X)=\alpha \lambda^{2} \gamma^{2 / \beta} \Gamma\left(1-\frac{2}{\beta}\right)\left[1+\sum_{i=1}^{\infty} \frac{a_{i}}{(i+1)^{1-(2 / \beta)}}\right] \\
& -\alpha^{2} \lambda^{2} \gamma^{2 / \beta}\left[\Gamma\left(1-\frac{1}{\beta}\right)\left\{1+\sum_{i=1}^{\infty} \frac{a_{i}}{(i+1)^{1-(k / \beta)}}\right\}\right]^{2} \\
& =\alpha \lambda^{2} \gamma^{2 / \beta}\left\{\Gamma\left(1-\frac{2}{\beta}\right)\left[1+\sum_{i=1}^{\infty} \frac{a_{i}}{(i+1)^{1-(2 / \beta)}}\right]\right. \\
& -\alpha\left[\Gamma\left(1-\frac{1}{\beta}\right)\right. \\
& \left.\left.\times\left\{1+\sum_{i=1}^{\infty} \frac{a_{i}}{(i+1)^{1-(k / \beta)}}\right\}\right]^{2}\right\} \text {. }
\end{aligned}
$$

\section{Mixture of Two GIGW (MGIGW) Distributions and Properties}

Everitt and Hand [15], Maclachlan and Krishnan [16], Maclachlan and Peel [17], Al-Hussaini and Sultan [18], Jiang et al. [19], and Sultan et al. [20] discussed various mixture distributions and found them useful in complex problems. The density of mixture of two GIGW distributions called Mixed Generalized Inverse Generalized Weibull (MGIGW) distribution is given by

$$
f_{\text {MGIGW }}(x ; \boldsymbol{\theta})=\sum_{j=1}^{2} p_{j} f_{j}\left(x, \boldsymbol{\theta}_{\mathbf{j}}\right),
$$

where for $j=1$ and 2

(i) $\boldsymbol{\theta}_{j}=\left(\lambda_{j}, \alpha_{j}, \beta_{j}, \gamma_{j}\right)^{T}$;

(ii) $f_{j}\left(x, \boldsymbol{\theta}_{j}\right)$ corresponds to $j$ th component of the mixture and is given by (7);

(iii) $\boldsymbol{\theta}=\left(\boldsymbol{\theta}_{1}^{T}, \boldsymbol{\theta}_{2}^{T}\right)^{T}$ and $\sum_{j=1}^{2} p_{j}=1$.

The density function of MGIGW distribution is plotted in Figure 4 for some combinations of values of $\gamma_{1}$ and $\gamma_{2}$ with $\lambda_{1}=6, \alpha_{1}=4$, and $\beta_{1}=0.5$ and $\lambda_{2}=3, \alpha_{2}=0.8$, and $\beta_{2}=3$. 


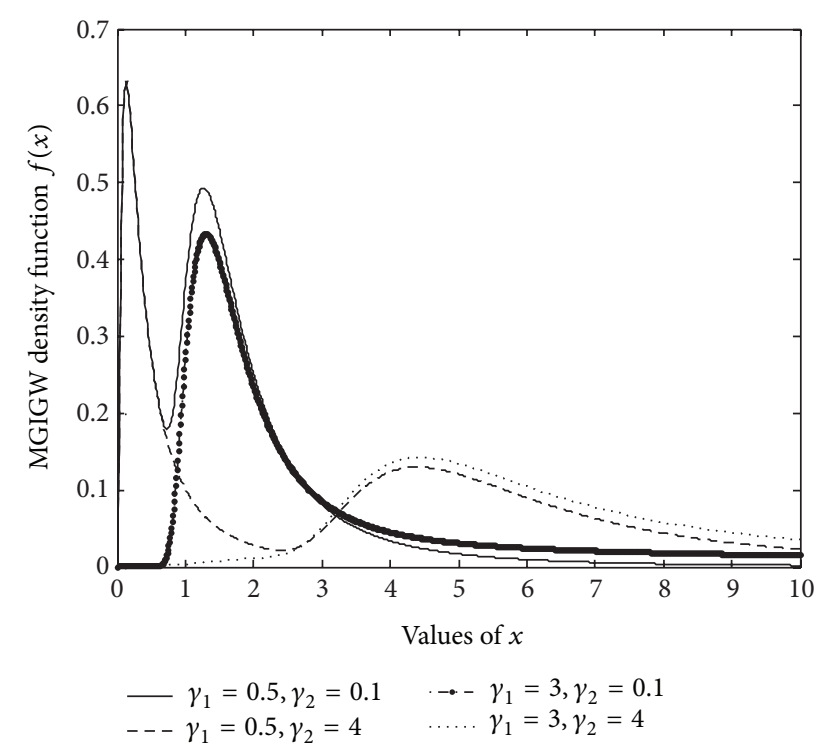

Figure 4: MGIGW density plots.

The corresponding cdf is given by

$$
F_{\text {MGIGW }}(x ; \boldsymbol{\theta})=\sum_{j=1}^{2} p_{j} F_{j}\left(x, \boldsymbol{\theta}_{j}\right),
$$

where $F_{j}\left(x, \boldsymbol{\theta}_{j}\right)$ is the cdf corresponding to $f_{j}\left(x, \boldsymbol{\theta}_{j}\right)$ for $j=1$ and 2.

3.1. Properties of MGIGW Distribution. The corresponding survival and failure rate functions are

$$
\begin{aligned}
\bar{F}_{\mathrm{MGIGW}}( & (x ; \boldsymbol{\theta})=\sum_{j=1}^{2} p_{j}\left(1-e^{-\gamma_{j}\left(\lambda_{j} / x\right)^{\beta_{j}}}\right)^{\alpha_{j}}, \\
h_{\mathrm{MGIGW}}(x)= & \left(\sum_{j=1}^{2} p_{j} \alpha_{j} \lambda_{j}^{\beta_{j}} \beta_{j} \gamma_{j} x^{-\left(\beta_{j}+1\right)} e^{-\gamma_{j}\left(\lambda_{j} / x\right)^{\beta_{j}}}\right. \\
& \left.\times\left(1-e^{-\gamma_{j}\left(\lambda_{j} / x\right)^{\beta_{j}}}\right)^{\alpha_{j}-1}\right) \\
& \times\left(\sum_{j=1}^{2} p_{j}\left(1-e^{-\gamma_{j}\left(\lambda_{j} / x\right)^{\beta_{j}}}\right)^{\alpha_{j}}\right)^{-1}
\end{aligned}
$$

respectively.

The $k$ th moment of MGIGW distribution can be written as

$$
\begin{aligned}
E\left(X^{k}\right)= & \sum_{j=1}^{2} p_{j} \lambda_{j}^{k} \gamma_{j}^{k / \beta_{j}} \alpha_{j} \Gamma\left(1-\frac{k}{\beta_{j}}\right) \\
& \times\left[1+\sum_{i=1}^{\infty} \frac{a_{i}}{(i+1)^{\left(1-k / \beta_{j}\right)}}\right] .
\end{aligned}
$$

Remark 3. For $\alpha_{j}=1$, the results are the same as those derived by de Gusmão et al. [14] for GIW.
3.2. Identifiability. Let $\Phi=\left\{F_{1}, F_{2}\right\}$ be a family of cdfs with transforms $\phi_{1}(t)$ and $\phi_{2}(t)$ for $t \in D_{\phi_{i}}$ (the domain of definition) such that the mapping $M: F_{i} \rightarrow \phi_{i}$ is linear and one-to-one. If there exists a total ordering $(\preceq)$ of $\Phi$ such that $F_{1} \preceq F_{2}$ implies

(i) $D_{\phi_{1}} \subseteq D_{\phi_{2}}$,

(ii) the existence of some $t_{1} \in D_{\phi_{1}}$ ( $t_{1}$ being independent of $\left.\phi_{2}\right)$ such that $\lim _{t \rightarrow t_{1}} \phi_{2}(t) / \phi_{1}(t)=0$,

then the class $\Phi^{\prime}$ of all finite mixtures of $\Phi$ is identifiable [21].

Using Chandra's approach [22] and the results by Sultan et al. [20], we prove the following proposition.

Proposition 4. The class of all finite mixing distributions relative to the GIGW distribution is identifiable.

Proof. If $X_{i}$ follows $\operatorname{GIGW}(\lambda, \alpha, \beta, \gamma)$ distribution, the $t$ th moment is

$$
\begin{aligned}
& \phi_{i}(t)=E\left(X_{i}^{t}\right) \\
&=\alpha_{i} \lambda_{i}^{t} \gamma_{i}^{t / \beta_{i}} \Gamma\left(1-\frac{t}{\beta_{i}}\right)\left[1+\sum_{l=1}^{\infty} \frac{a_{l}}{(l+1)^{1-\left(t / \beta_{i}\right)}}\right], \\
& i=1,2 .
\end{aligned}
$$

Using the cdf of $X_{i}$, we have

$F_{1}<F_{2}$ when $\beta_{1}=\beta_{2}, \lambda_{1}=\lambda_{2}, \gamma_{1}=\gamma_{2}, \alpha_{1}<\alpha_{2}$, $F_{1}<F_{2}$ when $\alpha_{1}<\alpha_{2}, \lambda_{1}=\lambda_{2}, \gamma_{1}=\gamma_{2}, \beta_{1}<\beta_{2}$.

Let $D_{\phi_{1}}(t)=\left(-\infty, \beta_{1}\right), D_{\phi_{2}}(t)=\left(-\infty, \beta_{2}\right)$, and $t=\beta_{1}$.

From (24), we have

$$
\begin{aligned}
\lim _{t \rightarrow \beta_{1}} \phi_{1}(t)= & \alpha_{1} \lambda_{1}^{t} \gamma_{1}^{t / \beta_{1}} \Gamma\left(1-\frac{t}{\beta_{1}}\right) \\
\times & \left\{1+\sum_{l=1}^{\infty} a_{l}(l+1)^{\left(t / \beta_{1}\right)-1}\right\}=\infty, \\
\lim _{t \rightarrow \beta_{1}} \phi_{2}(t)= & \alpha_{2} \lambda_{2}^{t} \gamma_{2}^{t / \beta_{2}} \Gamma\left(1-\frac{t}{\beta_{2}}\right) \\
& \times\left\{1+\sum_{l=1}^{\infty} a_{l}(l+1)^{\left(t / \beta_{2}\right)-1}\right\} \\
= & \alpha_{2} \lambda_{2}^{\beta_{1}} \gamma_{2}^{\beta_{1} / \beta_{2}} \Gamma\left(1-\frac{\beta_{1}}{\beta_{2}}\right) \\
& \times\left\{1+\sum_{l=1}^{\infty}(l+1)^{\left(\beta_{1} / \beta_{2}\right)-1}\right\} .
\end{aligned}
$$

Hence

$$
\begin{array}{r}
\lim _{t \rightarrow \beta_{1}} \frac{\phi_{2}(t)}{\phi_{1}(t)}=0 \quad \text { when } \lambda_{1}=\lambda_{2}<x, \gamma_{1}=\gamma_{2}, \\
\alpha_{1}=\alpha_{2}, \beta_{1}=\beta_{2} .
\end{array}
$$

This proves the identifiability of MGIGW distribution. 
In the next section, the maximum likelihood estimation (MLE) of the parameters of GIGW distribution is explored.

\section{Estimation for GIGW Distribution}

Let $X \sim \operatorname{GIGW}(\lambda, \alpha, \beta, \gamma)$ and $\boldsymbol{\theta}=(\lambda, \alpha, \beta, \gamma)^{T}$ be the parameter vector.

The equations for finding maximum likelihood estimates (MLEs) of parameters of GIGW distribution have been derived in the following subsections for uncensored and censored case.

4.1. Uncensored Case. A complete data set without any missing observation is termed as uncensored.

The log likelihood for a single observation $x$ of $X$ can be written as

$$
\begin{aligned}
l= & l(\lambda, \alpha, \beta, \gamma) \\
= & \log \alpha+\log \gamma+\log \beta+\beta \log \lambda-(\beta+1) \log x \\
& -\gamma\left(\frac{\lambda}{x}\right)^{\beta}+(\alpha-1) \log \left(1-e^{-\gamma(\lambda / x)^{\beta}}\right), \quad x>0 .
\end{aligned}
$$

The unit score vector is given by $\mathbf{U}=(\partial l / \partial \lambda, \partial l / \partial \alpha, \partial l / \partial \beta$, $\partial l / \partial \gamma)^{T}$.

Writing

$$
u=\left(1-e^{-\gamma(\lambda / x)^{\beta}}\right),
$$

we have

$$
\begin{aligned}
\frac{\partial l}{\partial \lambda}= & \frac{\beta}{\lambda}-\frac{\gamma \beta \lambda^{\beta-1}}{x^{\beta}}+\frac{(\alpha-1) e^{-\gamma(\lambda / x)^{\beta}} \beta \gamma \lambda^{\beta-1}}{\left(1-e^{-\gamma(\lambda / x)^{\beta}}\right) x^{\beta}} \\
= & \frac{\beta}{\lambda}-\frac{\gamma \beta \lambda^{\beta-1}}{x^{\beta}}+\frac{(\alpha-1) \beta \gamma \lambda^{\beta-1}(1-u)}{x^{\beta} u} ; \\
= & \frac{1}{\alpha}+\log \left(1-e^{-(\lambda / x)^{\beta}}\right)=\frac{1}{\alpha}+\log (u) ; \\
\frac{\partial l}{\partial \beta}= & \frac{1}{\beta}+\log \lambda-\log x \\
& +\frac{(\alpha-1) e^{-\gamma(\lambda / x)^{\beta}} \gamma(\lambda / x)^{\beta} \log (\lambda / x)}{\left(1-e^{-\gamma(\lambda / x)^{\beta}}\right)} \\
& -\gamma(\lambda / x)^{\beta} \log (\lambda / x) \\
= & \frac{1}{\beta}+\log \lambda-\log x \\
& +\frac{(\alpha-1) \gamma(\lambda / x)^{\beta} \log (\lambda / x)(1-u)}{u} \\
& -\gamma\left(\frac{\lambda}{x}\right)^{\beta} \log \left(\frac{\lambda}{x}\right)
\end{aligned}
$$

$$
\begin{aligned}
\frac{\partial l}{\partial \gamma} & =\frac{1}{\gamma}-\left(\frac{\lambda}{x}\right)^{\beta}+\frac{(\alpha-1) e^{-\gamma(\lambda / x)^{\beta}}(\lambda / x)^{\beta}}{\left(1-e^{-\gamma(\lambda / x)^{\beta}}\right)} \\
& =\frac{1}{\gamma}-\left(\frac{\lambda}{x}\right)^{\beta}+\frac{(\alpha-1)(\lambda / x)^{\beta}(1-u)}{(u)} .
\end{aligned}
$$

The MLE $\widehat{\boldsymbol{\theta}}$ of $\boldsymbol{\theta}$ can be obtained by solving the nonlinear equations

$$
\frac{\partial l}{\partial \lambda}=0, \quad \frac{\partial l}{\partial \alpha}=0, \quad \frac{\partial l}{\partial \beta}=0, \quad \frac{\partial l}{\partial \gamma}=0
$$

using numerical methods.

4.2. Censored Case. In survival analysis and reliability studies, we are generally encountered with censored data. Censoring occurs when the information about the survival time of the observations under study is incomplete. It represents a particular type of missing data [23]. Let $X_{i}$ and $C_{i}$ be independent random variables where $X_{i}$ is the lifetime of $i$ th individual and $C_{i}$ is the censoring time and $t_{i}=\min \left(X_{i}, C_{i}\right)$ for $i=1,2, \ldots, n$. The distribution of $C_{i}$ does not depend on any of the unknown parameters of $X_{i}$ where each $X_{i}$ follows GIGW distribution with $\boldsymbol{\theta}=(\lambda, \alpha, \beta, \gamma)^{T}$.

Let $r$ be the number of failures and let $F$ and $C$ denote the sets of uncensored and censored observations, respectively. The likelihood function for the censored case can be written as

$$
L=\prod_{i \in F} f\left(t_{i}\right) * \prod_{i \in C} \bar{F}\left(t_{i}\right),
$$

where $f\left(t_{i}\right)$ and $\bar{F}\left(t_{i}\right)$ are density and survival functions of GIGW distribution, respectively, and are given in (7) and (9).

Hence the censored log likelihood for GIGW distribution can be written as

$$
\begin{aligned}
l= & r\{\log \alpha+\log \gamma+\log \beta+\beta \log \lambda\} \\
& -(\beta+1) \sum_{i \in F} \log t_{i}-\gamma \lambda^{\beta} \sum_{i \in F} t_{i}^{-\beta} \\
& +(\alpha-1) \sum_{i \in F} \log \left(1-e^{-\gamma\left(\lambda / t_{i}\right)^{\beta}}\right) \\
& +\alpha \sum_{i \in C} \log \left(1-e^{-\gamma\left(\lambda / t_{i}\right)^{\beta}}\right) .
\end{aligned}
$$

For uncensored data, while writing the log likelihood function, the values of $x$ are from the complete sample but in case of censored data set, the log likelihood is split into two parts, one corresponding to censored observations and the other corresponding to those observations that are not censored.

Writing

$$
u_{i}=\left(1-e^{-\gamma\left(\lambda / t_{i}\right)^{\beta}}\right),
$$


the elements of the score vector $\mathbf{U}=(\partial l / \partial \lambda, \partial l / \partial \alpha, \partial l / \partial \beta$, $\partial l / \partial \gamma)^{T}$ are given by

$$
\begin{aligned}
\frac{\partial l}{\partial \lambda}= & \frac{r \beta}{\lambda}-\gamma \beta \lambda^{\beta-1} \sum_{i \in F} t_{i}^{\beta}+(\alpha-1) \beta \gamma \lambda^{\beta-1} \\
& \times \sum_{i \in F} t_{i}^{-\beta}\left(\frac{1-u_{i}}{u_{i}}\right)+\alpha \beta \gamma \lambda^{\beta-1} \sum_{i \in C} t_{i}^{-\beta}\left(\frac{1-u_{i}}{u_{i}}\right) ; \\
\frac{\partial l}{\partial \alpha}= & \frac{r}{\alpha}+\sum_{i \in F} \log \left(u_{i}\right) ; \\
\frac{\partial l}{\partial \beta}= & \frac{r}{\beta}+r \log \lambda-\sum_{i \in F} \log t_{i}-\gamma \lambda^{\beta} \sum_{i \in F} t_{i}^{-\beta} \log \left(\frac{\lambda}{t_{i}}\right) \\
& +(\alpha-1) \gamma \lambda^{\beta} \sum_{i \in F} t_{i}^{-\beta} \log \left(\frac{\lambda}{t_{i}}\right)\left(\frac{1-u_{i}}{u_{i}}\right) \\
& +\alpha \gamma \lambda^{\beta} \sum_{i \in C} t_{i}^{-\beta} \log \left(\frac{\lambda}{t_{i}}\right)\left(\frac{1-u_{i}}{u_{i}}\right) ; \\
\frac{\partial l}{\partial \gamma}= & \frac{r}{\gamma}-\lambda^{\beta} \sum_{i \in F} t_{i}^{\beta}+(\alpha-1) \lambda^{\beta} \sum_{i \in F} t_{i}^{-\beta}\left(\frac{1-u_{i}}{u_{i}}\right) .
\end{aligned}
$$

The $4 \times 4$ observed information matrix for interval estimation and hypothesis testing for parameters in $\boldsymbol{\theta}$ is given by

$$
\mathbf{K}=\mathbf{K}(\boldsymbol{\theta})=-\left[\begin{array}{llll}
K_{\lambda, \lambda} & K_{\lambda, \alpha} & K_{\lambda, \beta} & K_{\lambda, \gamma} \\
& K_{\alpha, \alpha} & K_{\alpha, \beta} & K_{\alpha, \gamma} \\
& & K_{\beta, \beta} & K_{\beta, \gamma} \\
& & & K_{\gamma, \gamma}
\end{array}\right] .
$$

Note that $\mathbf{K}(\boldsymbol{\theta})$ is observed and not the expected information matrix because for writing the elements of the expected information matrix, the expressions turn out to be very complicated. The expressions for the elements of $\mathbf{K}$ are given in appendix for censored case. The elements of $K$ for uncensored case can be analogously written using (27).

Under certain regularity conditions (fulfilled for parameters in the interior of the parameter space but not on the boundary),

$$
\sqrt{n}(\widehat{\boldsymbol{\theta}}-\boldsymbol{\theta}) \text { follows } N_{4}\left(0, \mathbf{I}(\boldsymbol{\theta})^{-1}\right) \text { asymptotically. }
$$

$\mathbf{I}(\boldsymbol{\theta})$ is the expected information matrix used for construction of tests of hypotheses and appropriate confidence regions for the parameters and can be replaced by the observed information matrix $\mathbf{K}(\widehat{\boldsymbol{\theta}})$. The asymptotic normality is useful for testing goodness of fit of GIGW distribution versus some of its submodels.

\section{Simulations and Application}

5.1. Estimation Based on Simulations. For checking the theoretical results given in Section 4, we simulate data by
TABLE 1: Estimates and RMSEs for parameters of GIGW.

\begin{tabular}{cccc}
\hline$n$ & Parameter & Estimate & RMSE \\
\hline \multirow{3}{*}{50} & $\lambda$ & .0528 & .0191 \\
& $\alpha$ & 11.4180 & 17.8873 \\
& $\beta$ & 2.3000 & 1.3033 \\
& $\gamma$ & .3692 & .2687 \\
\hline \multirow{3}{*}{100} & $\lambda$ & .0525 & .0132 \\
& $\alpha$ & 7.9380 & 10.0600 \\
& $\beta$ & 2.1150 & .6585 \\
& $\gamma$ & .3277 & .1824 \\
\hline \multirow{3}{*}{300} & $\lambda$ & .0505 & .0083 \\
& $\alpha$ & 6.2069 & 4.8450 \\
& $\beta$ & 2.0520 & .4325 \\
& $\gamma$ & .3228 & .1241 \\
\hline \multirow{3}{*}{300} & $\lambda$ & .0503 & .0064 \\
& $\alpha$ & 5.7614 & 3.2207 \\
& $\beta$ & 2.0289 & .3427 \\
& $\gamma$ & .3168 & .0987 \\
\hline
\end{tabular}

generating observations from GIGW distribution with $\lambda=$ $0.05, \alpha=5, \beta=2$, and $\gamma=0.3$. The assumed values for the parameters are arbitrary. The values of these parameters are estimated using quasi-Newton method in $R$. The sample sizes considered are $n=50,100,200$, and 300 with number of repetitions as 10,000, a standard followed in literature for carrying out simulations. The estimates of parameters with corresponding root mean square errors (RMSEs) are given in Table 1.

It is observed from Table 1 that estimates get closer to assumed values of parameters as sample size increases. It has also been checked that if we change the assumed values of parameters, the results will not vary. We consider another set of parametric values as $\lambda=3, \alpha=0.5, \beta=4$, and $\gamma=7$ and the results are reported in Table 2 .

It is observed from the values in Table 2 that, even on assuming different parametric values, the conclusions are similar to those drawn from Table 1.

5.2. Real Data Illustration. To check the superiority of GIGW distribution over GIW and IGW distributions, we use the censored data discussed in Sickle-Santanello et al. [24] and given in Klein and Moeschberger [23]. The data consist of death times (in weeks) of patients with cancer of tongue with aneuploid DNA profile. The observations are 1, 3, 3, 4, 10, 13, $13,16,16,24,26,27,28,30,30,32,41,51,61^{*}, 65,67,70,72$, $73,74^{*}, 77,79^{*}, 80^{*}, 81^{*}, 87^{*}, 87^{*}, 88^{*}, 89^{*}, 91,93,93^{*}, 96$, $97^{*}, 100,101^{*}, 104,104^{*}, 108^{*}, 109^{*}, 120^{*}, 131^{*}, 150^{*}, 157$, $167,231^{*}, 240^{*}$, and $400^{*}$, where asterisks denote censored observations.

For estimation purpose, the form of $l$ given by (32) is used with $r=31$. $C$ consists of observations with asterisks and other observations belong to the set $F$.

The MLEs for GIGW, GIW, and IGW distributions are given in Table 3 along with Akaike Information Criterion 
TABLE 2: Estimates and RMSEs for parameters of GIGW.

\begin{tabular}{cccc}
\hline$n$ & Parameter & Estimate & RMSE \\
\hline \multirow{3}{*}{50} & $\lambda$ & 3.0508 & .2889 \\
& $\alpha$ & .6286 & 1.0502 \\
& $\beta$ & 5.3610 & 3.6293 \\
& $\gamma$ & 7.9424 & 1.3347 \\
\hline \multirow{3}{*}{100} & $\lambda$ & 3.0319 & .22533 \\
& $\alpha$ & .5347 & .2836 \\
& $\beta$ & 4.5542 & 1.8225 \\
\multirow{3}{*}{200} & $\gamma$ & 7.6557 & 1.5891 \\
& $\lambda$ & 2.8116 & .2183 \\
& $\alpha$ & .5114 & .1649 \\
& $\beta$ & 4.2446 & 1.0356 \\
300 & $\gamma$ & 9.7055 & 2.9532 \\
\hline \multirow{3}{*}{30} & 2.975 & .15163 \\
& $\alpha$ & .5086 & .1266 \\
& $\alpha$ & 4.139 & .76085 \\
& $\gamma$ & 7.618 & 1.4384 \\
\hline
\end{tabular}

TABLE 3: Estimates of the parameters and the values of the AIC, BIC, and AICc.

\begin{tabular}{|c|c|c|c|c|}
\hline Model & Estimates & AIC & $\mathrm{BIC}$ & AICc \\
\hline \multirow{4}{*}{ GIGW } & $\widehat{\lambda}=5.88$ & \multirow{4}{*}{372.423} & \multirow{4}{*}{380.228} & \multirow{4}{*}{368.192} \\
\hline & $\widehat{\alpha}=86.87$ & & & \\
\hline & $\beta=.151$ & & & \\
\hline & $\widehat{\gamma}=7.27$ & & & \\
\hline \multirow{4}{*}{ GIW } & $\widehat{\lambda}=1.589$ & \multirow{4}{*}{375.281} & \multirow{4}{*}{381.134} & \multirow{4}{*}{371.742} \\
\hline & $\widehat{\alpha}=1.000$ & & & \\
\hline & $\widehat{\beta}=.489$ & & & \\
\hline & $\widehat{\gamma}=5.081$ & & & \\
\hline \multirow{4}{*}{ IGW } & $\widehat{\lambda}=13.679$ & \multirow{4}{*}{396.049} & \multirow{4}{*}{401.902} & \multirow{4}{*}{392.51} \\
\hline & $\widehat{\alpha}=1.183$ & & & \\
\hline & $\widehat{\beta}=.4216$ & & & \\
\hline & $\widehat{\gamma}=1$ & & & \\
\hline
\end{tabular}

(AIC), Bayesian Information Criterion (BIC), and Second Order Akaike Information Criterion (AICc).

It is observed from Table 3 that AIC, BIC, and AICc are lowest in case of GIGW distribution. So, we can conclude that GIGW distribution performs better than GIW and IGW distributions.

\section{Conclusions}

We introduce a new distribution named as Generalized Inverse Generalized Weibull (GIGW) distribution which is a generalization of Generalized Inverse Weibull (GIW) distribution and Inverse Generalized Weibull (IGW) distribution. Mathematical properties of GIGW have been discussed. The theory to find maximum likelihood estimates of GIGW distribution for complete and censored data has also been provided. The applicability of the proposed model and its superiority over some of other models have been established using a real data set.

\section{Appendix}

The expressions for the elements of $\mathbf{K}$ for censored case are given below:

$$
\begin{aligned}
K_{\lambda, \lambda}= & \frac{\partial^{2} l}{\partial \lambda^{2}} \\
= & \frac{-r \beta}{\lambda^{2}}-\gamma \beta(\beta-1) \lambda^{\beta-2} \sum_{i \in F} t_{i}^{-\beta}+(\alpha-1) \gamma \beta \lambda^{\beta-2} \\
& \times \sum_{i \in F} t_{i}^{-\beta}\left(\frac{1-u_{i}}{u_{i}}\right)\left[\beta-1-\frac{\gamma \beta}{u_{i}}\left(\frac{\lambda}{t_{i}}\right)^{\beta}\right]+\alpha \gamma \beta \lambda^{\beta-2} \\
& \times \sum_{i \in C} t_{i}^{-\beta}\left(\frac{1-u_{i}}{u_{i}}\right)\left[\beta-1-\frac{\gamma \beta}{u_{i}}\left(\frac{\lambda}{t_{i}}\right)^{\beta}\right] ;
\end{aligned}
$$$$
K_{\lambda, \alpha}=\frac{\partial^{2} l}{\partial \lambda \partial \alpha}
$$$$
=\gamma \beta \lambda^{\beta-1} \sum_{i \in F} t_{i}^{-\beta}\left(\frac{1-u_{i}}{u_{i}}\right)
$$$$
+\gamma \beta \lambda^{\beta-1} \sum_{i \in C} t_{i}^{-\beta}\left(\frac{1-u_{i}}{u_{i}}\right)
$$$$
K_{\lambda, \beta}=\frac{\partial^{2} l}{\partial \lambda \partial \beta}
$$$$
\begin{gathered}
=\frac{r}{\lambda}-\gamma \lambda^{\beta-1} \sum_{i \in F} t_{i}^{-\beta}\left\{1+\beta \log \left(\frac{\lambda}{t_{i}}\right)\right\} \\
+(\alpha-1) \gamma \lambda^{\beta-1} \sum_{i \in F} t_{i}^{-\beta}\left(\frac{1-u_{i}}{u_{i}}\right)
\end{gathered}
$$$$
\times\left[1+\beta \log \left(\frac{\lambda}{t_{i}}\right)\left\{1-\frac{\gamma}{u_{i}}\left(\frac{\lambda}{t_{i}}\right)^{\beta}\right\}\right]
$$$$
+\alpha \gamma \lambda^{\beta-1} \sum_{i \in C} t_{i}^{-\beta}\left(\frac{1-u_{i}}{u_{i}}\right)
$$$$
\times\left[1+\beta \log \left(\frac{\lambda}{t_{i}}\right)\left\{1-\frac{\gamma}{u_{i}}\left(\frac{\lambda}{t_{i}}\right)^{\beta}\right\}\right]
$$

$$
\begin{aligned}
K_{\lambda, \gamma}= & \frac{\partial^{2} l}{\partial \lambda \partial \gamma} \\
= & -\beta \lambda^{\beta-1} \sum_{i \in F} t_{i}^{-\beta}+(\alpha-1) \beta \lambda^{\beta-1} \\
& \times \sum_{i \in F} t_{i}^{-\beta}\left(\frac{1-u_{i}}{u_{i}}\right)\left[1-\frac{\gamma}{u_{i}}\left(\frac{\lambda}{t_{i}}\right)^{\beta}\right] \\
& +\alpha \beta \lambda^{\beta-1} \sum_{i \in C} t_{i}^{-\beta}\left(\frac{1-u_{i}}{u_{i}}\right)\left[1-\frac{\gamma}{u_{i}}\left(\frac{\lambda}{t_{i}}\right)^{\beta}\right] ;
\end{aligned}
$$




$$
\begin{aligned}
& K_{\alpha, \alpha}=\frac{\partial^{2} l}{\partial \alpha^{2}}=\frac{-r}{\alpha^{2}} ; \\
& K_{\alpha, \beta}=\frac{\partial^{2} l}{\partial \alpha \partial \beta}=\gamma \lambda^{\beta} \sum_{i \in F} t_{i}^{-\beta}\left(\frac{1-u_{i}}{u_{i}}\right) \log \left(\frac{\lambda}{t_{i}}\right) ; \\
& K_{\alpha, \gamma}=\frac{\partial^{2} l}{\partial \alpha \partial \gamma}=\lambda^{\beta} \sum_{i \in F} t_{i}^{-\beta}\left(\frac{1-u_{i}}{u_{i}}\right) ; \\
& K_{\beta, \beta}=\frac{\partial^{2} l}{\partial \beta^{2}} \\
& =\frac{-r \beta}{\lambda^{2}}-\gamma \lambda^{\beta} \sum_{i \in F} \log \left(\frac{\lambda}{t_{i}}\right)\left[t_{i}^{-\beta} \log \left(\frac{\lambda}{t_{i}}\right)\right] \\
& +(\alpha-1) \gamma \lambda^{\beta} \sum_{i \in F} \log \left(\frac{\lambda}{t_{i}}\right)\left(\frac{1-u_{i}}{u_{i}}\right) \\
& \times\left[t_{i}^{-\beta} \log \left(\frac{\lambda}{t_{i}}\right)-\frac{\gamma t_{i}^{-\beta}}{u_{i}}\left(\frac{\lambda}{t_{i}}\right)^{\beta} \log \left(\frac{\lambda}{t_{i}}\right)\right] \\
& +\alpha \gamma \lambda^{\beta} \sum_{i \in C} \log \left(\frac{\lambda}{t_{i}}\right)\left(\frac{1-u_{i}}{u_{i}}\right) \\
& \times\left[t_{i}^{-\beta} \log \left(\frac{\lambda}{t_{i}}\right)-\frac{\gamma t_{i}^{-\beta}}{u_{i}}\left(\frac{\lambda}{t_{i}}\right)^{\beta} \log \left(\frac{\lambda}{t_{i}}\right)\right] ; \\
& K_{\beta, \gamma}=\frac{\partial^{2} l}{\partial \beta \partial \gamma} \\
& =-\lambda^{\beta} \sum_{i \in F} t_{i}^{-\beta} \log \left(\frac{\lambda}{t_{i}}\right)+(\alpha-1) \lambda^{\beta} \\
& \times \sum_{i \in F} t_{i}^{-\beta} \log \left(\frac{\lambda}{t_{i}}\right)\left(\frac{1-u_{i}}{u_{i}}\right)\left[1-\frac{\gamma}{u_{i}}\left(\frac{\lambda}{t_{i}}\right)^{\beta}\right] \\
& +\alpha \lambda^{\beta} \sum_{i \in C} t_{i}^{-\beta} \log \left(\frac{\lambda}{t_{i}}\right)\left(\frac{1-u_{i}}{u_{i}}\right) \\
& \times\left[1-\frac{\gamma}{u_{i}}\left(\frac{\lambda}{t_{i}}\right)^{\beta}\right] \\
& \begin{aligned}
K_{\gamma, \gamma}= & \frac{\partial^{2} l}{\partial \gamma^{2}} \\
= & \frac{-r}{\gamma^{2}}-(\alpha-1) \lambda^{2 \beta} \sum_{i \in F} t_{i}^{-2 \beta}\left(\frac{1-u_{i}}{u_{i}^{2}}\right) \\
& -\alpha \lambda^{2 \beta} \sum_{i \in C} t_{i}^{-2 \beta}\left(\frac{1-u_{i}}{u_{i}^{2}}\right) .
\end{aligned}
\end{aligned}
$$

\section{Conflict of Interests}

The authors declare that there is no conflict of interests regarding the publication of this paper.

\section{Acknowledgment}

The second author is grateful to University Grants Commission, Government of India, for providing financial support for this work.

\section{References}

[1] G. S. Mudholkar and D. K. Srivastava, "Exponentiated Weibull family for analyzing bathtub failure-rate data," IEEE Transactions on Reliability, vol. 42, no. 2, pp. 299-302, 1993.

[2] G. S. Mudholkar, D. K. Srivastava, and M. Friemer, "The exponentiated weibull family: a reanalysis of the bus-motorfailure data," Technometrics, vol. 37, no. 4, pp. 436-445, 1995.

[3] G. S. Mudholkar and A. D. Hutson, "The exponentiated weibull family: some properties and a flood data application," Communications in Statistics: Theory and Methods, vol. 25, no. 12, pp. 3059-3083, 1996.

[4] R. G. Voda, "On the inverse rayleigh variable," Union of Japanese Scientists and Engineers, vol. 19, no. 4, pp. 15-21, 1972.

[5] P. Erto and M. Rapone, "Non-informative and practical Bayesian confidence bounds for reliable life in the Weibull model," Reliability Engineering, vol. 7, no. 3, pp. 181-191, 1984.

[6] A. Drapella, "ComplementaryWeibull distribution: unknown or just forgotten," Quality and Reliability Engineering International, vol. 9, pp. 383-385, 1993.

[7] R. Johnson, S. Kotz, and N. Balakrishnan, Continuous Univariate Distributions, Wiley-Interscience, New York, NY, USA, 2nd edition, 1995.

[8] P. Pawlas and D. Szynal, "Characterizations of the inverse Weibull distribution and generalized extreme value distributions by moments of $\mathrm{k}$ th record values," Applicationes Mathematicae, vol. 27, no. 2, pp. 197-202, 2000.

[9] A. Z. Keller, A. R. R. Kamath, and U. D. Perera, "Reliability analysis of CNC machine tools," Reliability Engineering, vol. 3, no. 6, pp. 449-473, 1982.

[10] G. S. Mudholkar and G. D. Kolia, "Generalized Weibull family: a structural analysis," Communications in Statistics Series A: Theory and Methods, vol. 23, pp. 1149-1171, 1994.

[11] D. N. P. Murthy, M. Xie, and R. Jiang, Weibull Models, John Wiley \& Sons, New York, NY, USA, 2004.

[12] R. Calabria and G. Pulcini, "Bayes 2-sample prediction for the inverse weibull distribution," Communications in StatisticsTheory and Methods, vol. 23, no. 6, pp. 1811-1824, 1994.

[13] M. Aleem and G. R. Pasha, "Ratio, product and single moments of order statistics from inverse Weibull distribution," Journal of Statistics, vol. 10, no. 1, pp. 1-7, 2003.

[14] F. R. S. de Gusmão, E. M. M. Ortega, and G. M. Cordeiro, "The generalized inverse Weibull distribution," Statistical Papers, vol. 52, no. 3, pp. 591-619, 2011.

[15] B. S. Everitt and D. J. Hand, Finite Mixture Distributions, Chapman \& Hall, London, UK, 1981.

[16] G. J. Maclachlan and T. Krishnan, The EM Algorithm and Extensions, John Wiley \& Sons, New York, NY, USA, 1997.

[17] G. Maclachlan and D. Peel, Finite Mixture Models, John Wiley \& Sons, New York, NY, USA, 2000.

[18] E. K. AL-Hussaini and K. S. Sultan, "Reliability and hazard based on nite mixture models," in Handbook of Statistics, N. Balakrishnan and C. R. Rao, Eds., vol. 20, pp. 139-183, Elsevier, Amsterdam, The Netherlands, 2001. 
[19] R. Jiang, D. N. P. Murthy, and P. Ji, "Models involving two inverse Weibull distributions," Reliability Engineering and System Safety, vol. 73, no. 1, pp. 73-81, 2001.

[20] K. S. Sultan, M. A. Ismail, and A. S. Al-Moisheer, "Mixture of two inverse Weibull distributions: properties and estimation," Computational Statistics and Data Analysis, vol. 51, no. 11, pp. 5377-5387, 2007.

[21] H. Teicher, "Identifiability of finite mixtures," Annals of Mathematical Statistics, vol. 34, pp. 1265-1269, 1963.

[22] S. Chandra, "On the mixtures of probability distributions," Scandinavian Journal of Statistics, vol. 4, no. 3, pp. 105-112, 1977.

[23] J. P. Klein and M. L. Moeschberger, Survival Analysis: Techniques for Censored and Truncated Data, Springer, New York, NY, USA, 2003.

[24] B. J. Sickle-Santanello, W. B. Farrar, S. Keyhani-Rofagha et al., "A reproducible System of flow cytometric DNA analysis of paraffin embedded solid tumors: technical improvements and statistical analysis," Cytometry, vol. 9, pp. 594-599, 1988. 


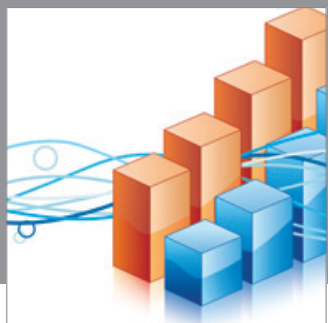

Advances in

Operations Research

mansans

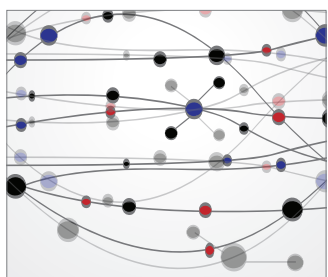

The Scientific World Journal
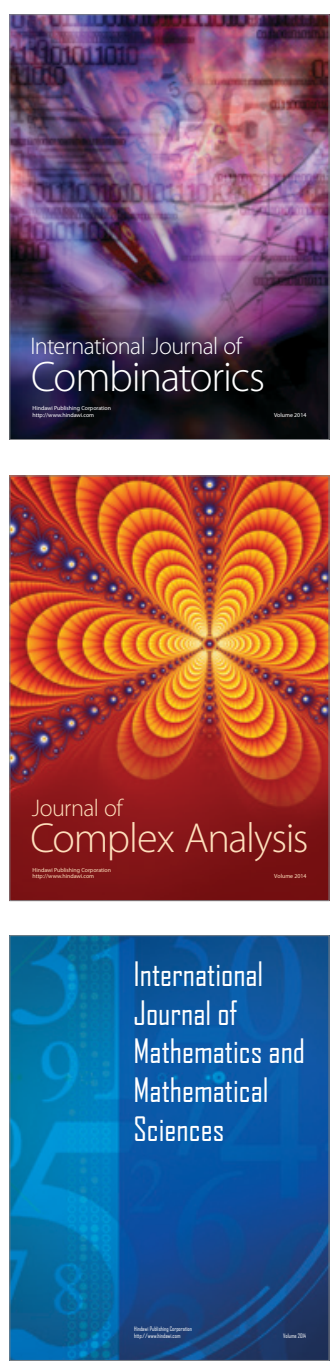
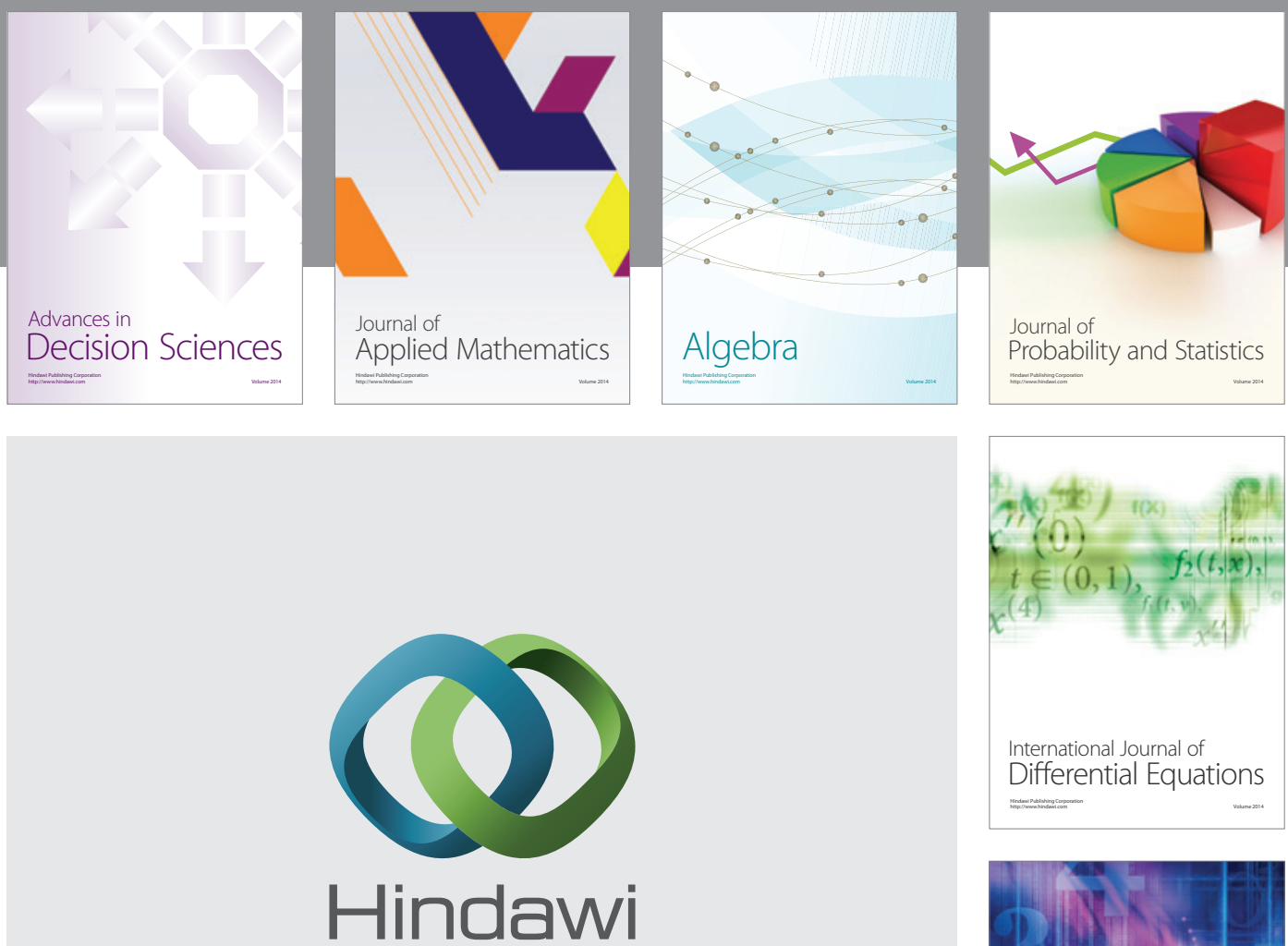

Submit your manuscripts at http://www.hindawi.com
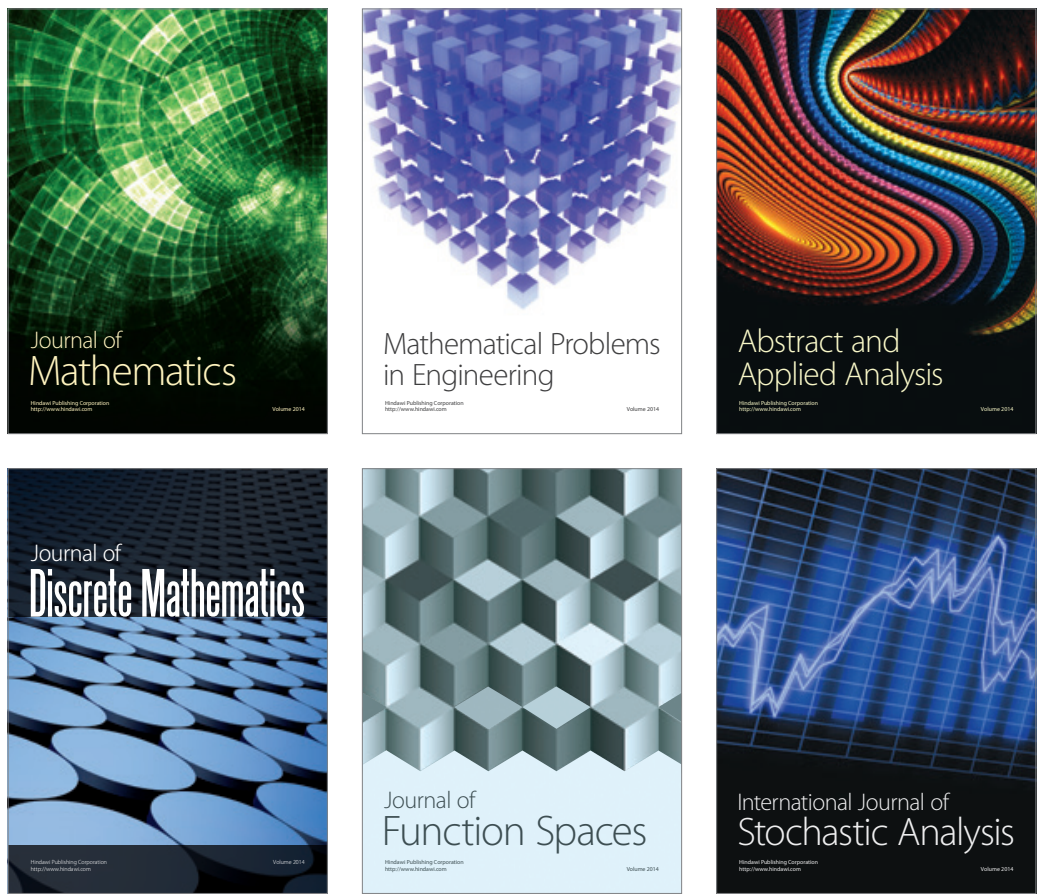

Journal of

Function Spaces

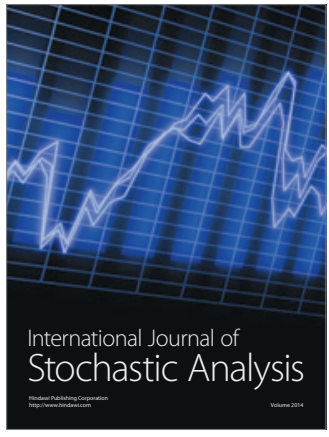

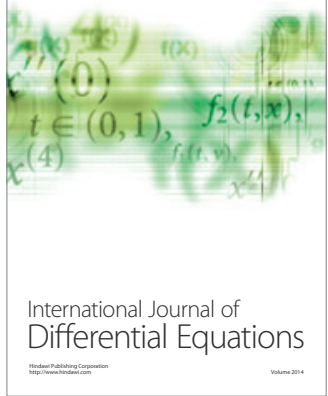
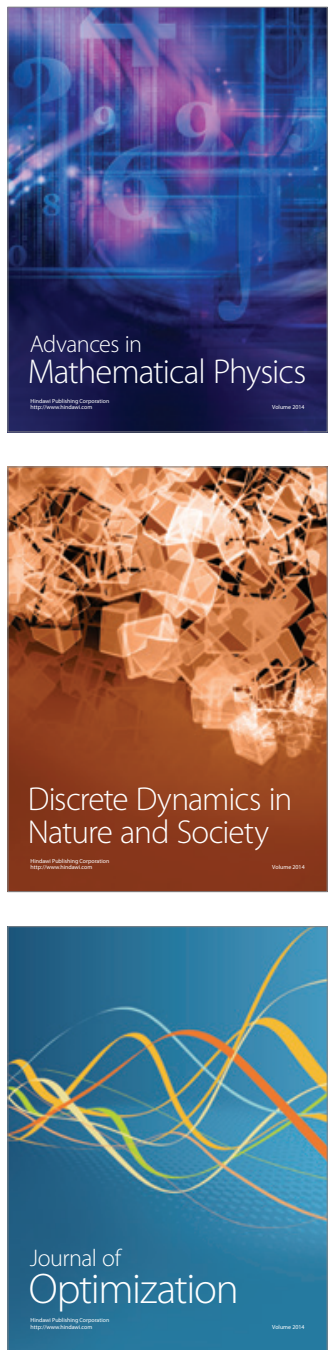